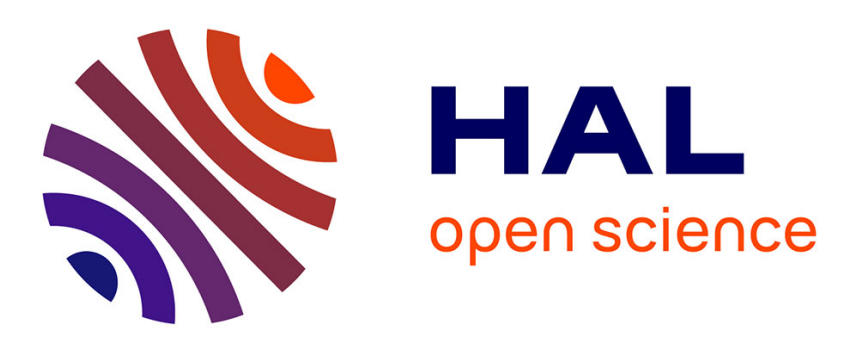

\title{
Slow light induced by Stimulated Raman Scattering on spatial Kerr soliton
}

Jeremy Michaud, Gil Fanjoux, Hervé Maillotte, Luca Furfaro, Thibaut

Sylvestre

\section{- To cite this version:}

Jeremy Michaud, Gil Fanjoux, Hervé Maillotte, Luca Furfaro, Thibaut Sylvestre. Slow light induced by Stimulated Raman Scattering on spatial Kerr soliton. Annales de Physique, 2007, 32 (2-3), pp.103106. hal-00272946

\section{HAL Id: hal-00272946 https://hal.science/hal-00272946}

Submitted on 29 Aug 2013

HAL is a multi-disciplinary open access archive for the deposit and dissemination of scientific research documents, whether they are published or not. The documents may come from teaching and research institutions in France or abroad, or from public or private research centers.
L'archive ouverte pluridisciplinaire HAL, est destinée au dépôt et à la diffusion de documents scientifiques de niveau recherche, publiés ou non, émanant des établissements d'enseignement et de recherche français ou étrangers, des laboratoires publics ou privés. 


\title{
Slow light induced by stimulated Raman scattering on spatial Kerr soliton
}

\author{
J. Michaud ${ }^{\text {a }}$, G. Fanjoux, H. Maillotte, L. Furfaro and T. Sylvestre \\ Département d'Optique P.M. Duffieux, Institut FEMTO-ST, Université de Franche- \\ Comté, CNRS 6174, 25030 Besançon, France
}

\begin{abstract}
We present numerical and experimental results demonstrating the slow light effect induced by the sharp resonance of the Raman gain on a spatial Raman soliton in a Kerr planar waveguide.
\end{abstract}

\section{INTRODUCTION}

It is now possible to generate large optical delay of a signal pulse by generating at the signal frequency a strong dispersion associated with a laser-induced amplifying resonance, such as that arising from stimulated Raman or Brillouin scattering $[1,2]$ (for more details about slow light see Ref. [3] and ref therein). Furthermore, if the nonlinear medium has a strong self-focusing nonlinearity, in this way it becomes possible to generate slow-light spatial solitons. Based on this idea, this work demonstrates both numerically and experimentally, for the first time to our knowledge, the slow light process induced by Stimulated Raman Scattering (SRS) on a spatial soliton [4]. This is achieved in a $\mathrm{CS}_{2}$ nonlinear planar waveguide that possesses both a strong self-focusing nonlinearity to generate spatial Kerr soliton and a Raman susceptibility sharp enough to induce the slow light process simultaneously.

\section{NUMERICAL RESULTS}

We developed a spatiotemporal numerical model based on the nonlinear Schrödinger equation including the group velocity dispersion, diffraction in the free transverse dimension $x$ of the planar waveguide, the instantaneous Kerr effect and the delayed Raman response. In our case, we consider a narrow gain band

\footnotetext{
${ }^{a}$ e-mail: jmichaud@univ-fcomte.fr
} 
Raman response with a real part which induces to a sharp transition in the effective refractive index of the material. Indeed, the narrow gain band of $\mathrm{CS}_{2}$ of $15 \mathrm{GHz}$ allows for the achievement of strong group index changes at the Raman phonon frequency.

Figures $1 \mathrm{a}$ and $1 \mathrm{~b}$ illustrate the longitudinal evolution of the normalized spaceintegrated temporal profiles for the pump and the Stokes pulses, respectively. While the pump pulse stays unchanged till $1.7 \mathrm{~cm}$, i.e., before the trailing edge depletion (Fig. 1a) due to the strong conversion regime, the Raman pulse evolves with three distinct steps. First, it is generated from quantum noise at the top of the pump pulse where the Raman gain is the most important. Thereafter, it undergoes a large optical delay by slowing down toward the trailing edge of the pump, up to the pump depletion regime. This delay, opposite and well greater than the walk-off due to group velocity mismatch (GVM), is unambiguously due to the slow light process. Finally, the slow light process is annihilated by the pump depletion, and the Raman pulse moves forward the pump leading edge, where the Raman gain still exists.

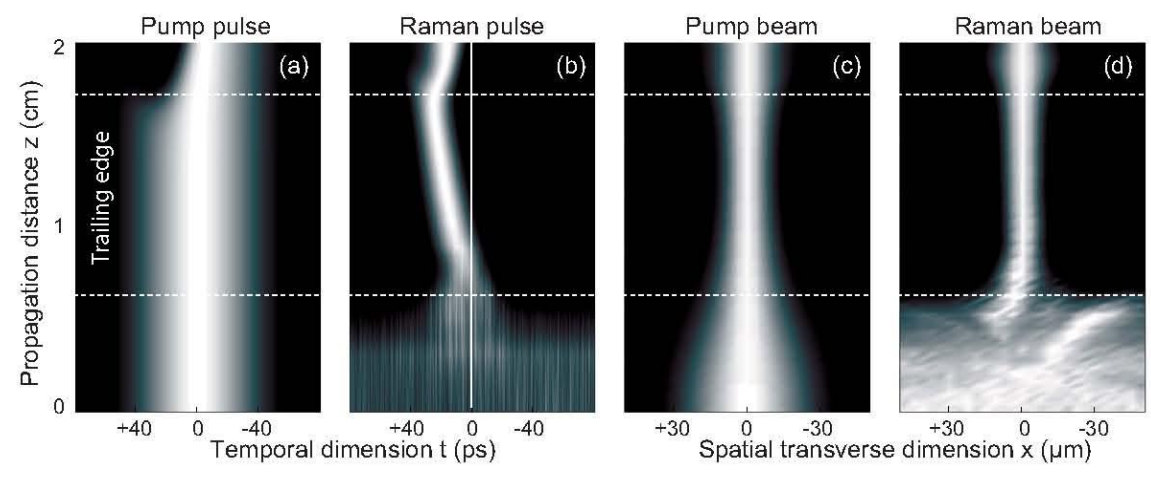

Figure 1. Numerical results: longitudinal evolution of the normalized temporal and spatial profiles of (a-c) the pump and (b-d) the Raman Stokes component in the $2 \mathrm{~cm}$ long planar waveguide. $P_{i}=0.8 \mathrm{GW} / \mathrm{cm}^{2}$ (soliton power $P_{s}=0.25 \mathrm{GW} / \mathrm{cm}^{2}$ ). Other parameters are $\beta_{2}=+4.24 \times 10^{-25} \mathrm{~s}^{2} / \mathrm{m}, n_{2}=+3.5 \times 10^{-18} \mathrm{~m}^{2} / \mathrm{W}$ and $\lambda=532 \mathrm{~nm}$.

Concerning the spatial dynamics, Figures $1 \mathrm{c}$ and $1 \mathrm{~d}$ show the longitudinal evolution of the normalized time-integrated spatial profiles for the pump and the Stokes beams, respectively. Figure 1c shows that the pump beam first undergoes a strong self-focusing because the input power is greater than the soliton power. The Stokes beam, shown in Figure 1d, is spatially confined by the self-focused pump beam, due to both the Raman gain and to cross-phase modulation (XPM) [4]. It thus propagates with a stable spatial width till the depletion of the pump. Figures $1 \mathrm{~b}$ and $1 \mathrm{~d}$ together clearly demonstrate a slow-light spatial soliton, indicated by the two horizontal white dotted lines.

\section{EXPERIMENTAL RESULTS}

The experimental setup is made up of a $3 \mathrm{~cm}$ long Kerr planar waveguide made of thin liquid $\mathrm{CS}_{2}$ layer sandwiched between two SK5 glass blocks. The pump 
source is a powerchip frequency-doubled Nd:YAG laser $(1 \mathrm{kHz}, 380 \mathrm{ps}, 532 \mathrm{~mm}$, see Ref. [4] for more details). The input beam spatial width is $52 \mu \mathrm{m}$. The pump and the Stokes beams are spatially divided at the waveguide's output, end by means of a diffraction grating (2400 lines/mm) and measured both in time and in space by using a streak camers with a 5 ps resolution. Figures 2a-2c show spatiotemporal pump and Stokes beams at the waveguide's output for (a) the pump in diffraction regime, (b) the pump and (c) the corresponding Raman Stokes. Time-integrated spatial profiles are also shown in Figure 2 (bottom) and space-integrated temporal profiles (right side). By comparing Figures $2 \mathrm{a}$ and $2 \mathrm{~b}$, we obeerve that the pump presents a depletion of its trailing edge where the Raman Stokes (Fig. 2c) is clearly oberved, in agreement, with our numerical simulations. Moreover, it is noteworthy to observe that the experimental spatial profiles are perfectly fitted by the theoretical ones (dashed curves). The Stokes beam has a clear spatial soliton profile due to the fact, that it is only generated when the pump reached a soliton regime. Before the pump depletion, we have measured that the time delay of the Raman pulse is linearly dependent on the input power, in agrement, with the slow-light theory [5]. The maximum delay is about $120 \mathrm{ps}$ for $3 \mathrm{~cm}$ of propagation, leading to an equivalent group velocity equal to $/ 2.8$ for the Raman Stokes.
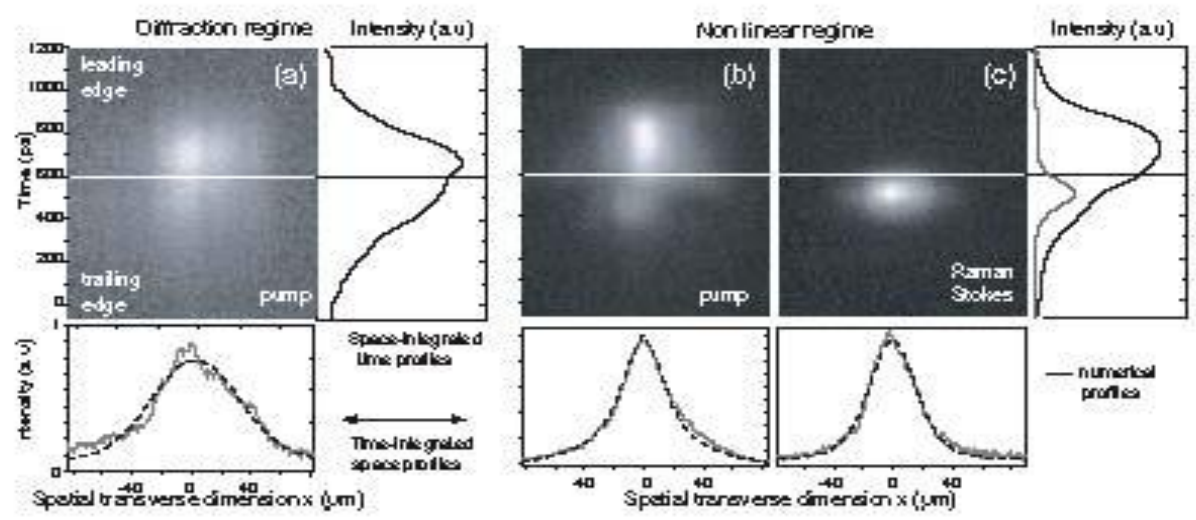

Figure 2. Experimental spatiotemporal profiles with time-integrated spatial profiles (bottom) and space-integrated temporal profiles (right side) at the waveguide's output for (a) the pump in diffraction regime ( $R=1 \mathrm{~mW}$ ), (b) the pump and (c) the Raman Stokes in nonlinear regime and at the end of the slow light st, age $(R=1.68 \mathrm{~mW})$. Soliton porer is $P_{s}=1.2 \mathrm{~mW}$.

\section{CONCLUSION}

We have demonstrated the slow-light effect, on a Kerr spatial soliton induced by stimulated Raman scattering in a nonlinear waveguide. The Raman Stokes pulse has a $120 \mathrm{ps}$ delay for $3 \mathrm{~cm}$ of propagation while preserving spatial soliton properties. 
To be published in Ann. Phys. Fr. 2007

\section{References}

[1] J.E. Sharping, Y. Okawachi, A.L. Gaeta, Opt. Exp. 13, 6092 (2005).

[2] K.Y. Song, M.G. Herraez, L. Thévanaz, Opt. Exp. 13, 82 (2005).

[3] R.W Boyd, D.J. Gauthier, in Progress in Optics 43 (Wolf Ed., Amsterdam, 2002).

[4] G. Fanjoux, J. Michaud, M. Delqué, H. Maillotte, T. Sylvestre, Opt. Lett. 31, 3480 (2006).

[5] K. Lee, N.M. Lawandy, App. Phys. Lett. 78, 704 (2001). 